\title{
CONCEPÇÃO DE UMA CÂMARA DE ENVELHECIMENTO ACELERADO COM USO DO ARDUINO
}

\author{
VAlmir Nascimento Júnior, Raphael Borges Nobrega, Ronimack Trajano Souza, \\ Higor COSTA Rocha \\ Universidade Federal de Campina Grande - UFCG \\ <valmir.junior@ee.ufcg.edu.br>.<raphael.nobrega@ee.ufcg.edu.br>.<ronimack@dee.ufcg.edu.br> \\ <higorcosta4@gmail.com> \\ 10.21439/conexoes.v15i0.2143
}

\begin{abstract}
Resumo. A aplicação de materiais poliméricos no desenvolvimento de produtos e no revestimento de equipamentos destinados a aplicações de uso externo tem aumentado significativamente com os avanços tecnológicos e na ciência dos materiais. No entanto, os polímeros são susceptíveis a degradação provocada pela ação dos diversos agentes presentes no meio ambiente e que podem ocasionar falhas prematuras em serviço. A realização de testes de envelhecimento acelerado em laboratório permite avaliar o comportamento dos materiais poliméricos em curtos períodos e detectar materiais com propriedades inadequadas ou susceptíveis a falhas. Um dos testes consiste na utilização de câmaras de envelhecimento acelerado que permitem submeter amostras do material polimérico à radiação ultravioleta, temperatura e umidade. No entanto, os equipamentos disponibilizados no mercado possuem valores elevados e que dificultam a aquisição pelos laboratórios de pesquisa. Neste contexto, o presente trabalho teve como objetivo o projeto e desenvolvimento de uma câmara de envelhecimento acelerado de baixo custo para a realização de ensaios com materiais poliméricos. O equipamento é constituído por lâmpadas ultravioleta e um sistema de condensação que permitem simular a exposição de amostras à radiação UV, temperatura e umidade. Os mecanismos podem ser selecionados e operados de forma cíclica a partir de um sistema de controle microcontrolado baseado na plataforma open-source Arduino.
\end{abstract}

Palavras-chaves: Câmara. Degradação. Envelhecimento. Polímeros. Radiação ultravioleta.

\section{DESIGN OF AN ACCELERATED AGING CHAMBER USING THE ARDUINO}

\begin{abstract}
The application of polymeric materials in product development and coating of equipment for outdoor applications has increased with advances in technology and materials science. However, polymers are susceptible to degradation caused by the action of various agents present in the environment and which may cause premature failures in service. Accelerated aging testing in the laboratory evaluates the behavior of polymeric materials over short periods and detects materials with inadequate or fail-safe properties. One of the tests is the use of accelerated aging chambers that allow samples of the polymeric material to be subjected to ultraviolet radiation, temperature and humidity. However, the equipment available in the market has high values that make it difficult for the research laboratories to acquire them. In this context, the present work aimed to design and develop a low cost accelerated aging chamber for testing polymeric materials. The equipment consists of ultraviolet lamps and a condensation system that allow simulating the exposure of samples to UV radiation, temperature and humidity. The mechanisms can be cyclically selected and operated from a microcontrolled control system based on the open-source Arduino platform.
\end{abstract}

Keywords: Aging. Chamber. Degradation. Polymers. Ultraviolet radiation.

\section{INTRODUÇÃO}

Atualmente, os materiais poliméricos têm ocupado uma parcela significativa em aplicações de uso externo em diversas áreas. No setor elétrico, os materiais poliméricos têm sido largamente utilizados para prover isolação elétrica, como no encapsulamento de condutores elétricos e na composição de isoladores utilizados em sistemas de média e alta tensão.

Apesar do uso generalizado dos produtos poliméricos, a susceptibilidade aos fenômenos de envelhecimento pode provocar a degradação do material e ocasionar a falha prematura em serviço (RABELLO, 2000). A degradação polimérica é causada por diversos tipos de reações químicas que ocorrem no material durante $o$ 
processamento ou uso final. Dentre os tipos de degradação, a oxidação representa uma das causas mais importantes para avaliação do desempenho do material polimérico (RABELLO, 2000; FECHINE; SANTOS; RABELLO, 2006). A oxidação pode ser iniciada por vários meios, principalmente por altas temperaturas (termooxidação) e por radiação ultravioleta (foto-oxidação).

Para avaliar o desempenho e obter estimativas do tempo de vida útil, os materiais poliméricos são submetidos a testes em laboratório com o objetivo de promover o processo degradativo do material de maneira acelerada e padronizada (NEKEB et al., 2012, NAZIR; PHUNG, 2016; ULLAH et al., 2020). A principal vantagem dos testes consiste na estimação da vida útil e obtenção de dados de forma prática com períodos relativamente curtos para condições semelhantes a real.

$\mathrm{O}$ envelhecimento provocado por exposição à radiação UV pode ser simulado em laboratório por meio da utilização de lâmpadas fluorescentes especiais ou de arco de xenônio, que emitem radiação na faixa espectral do ultravioleta e permite emular a incidência solar.

Em geral, os ensaios de envelhecimento artificial por radiação UV são realizados em câmaras de intemperismo que expõem os corpos de provas a ciclos alternados de radiação UV, umidade e temperatura. Os fatores que causam a degradação podem ser simulados isoladamente ou em conjunto, de forma controlada. No entanto, a aquisição do equipamento nem sempre é possível devido aos custos associados, podendo comprometer a realização de pesquisas na área. Além disso, a duração dos ensaios é geralmente superior a 1.000 horas, podendo gerar a indisponibilidade do equipamento para outros pesquisadores.

Uma alternativa para a realização de ensaios de envelhecimento por radiação UV consiste na construção do equipamento, uma vez que os componentes eletrônicos são acessíveis no mercado e os aspectos construtivos são normatizados. Os procedimentos para construção da câmara, exposição dos corpos de prova e parâmetros necessários para a realização do ensaio normalmente são baseados nas recomendações das normas (ASTM G. 53, 1998; ASTM G. 151, 2000, ASTM G. 154, 2016).

Neste contexto, Saron (2001) desenvolveu um protótipo que permite a instalação de até oito lâmpadas UV, subdivididas em dois compartimentos compostos por quatro lâmpadas para a realização de ensaios de amostras. O controle dos ciclos de exposição à radiação UV e condensação é realizado por meio de temporizadores.

O presente trabalho tem como objetivo o desenvolvimento de um equipamento automatizado e de baixo custo para simular em laboratório condições que levam à degradação do material polimérico em situações de uso prático quando expostos a radiação UV, temperatura e umidade. $\mathrm{O}$ controle dos ciclos de exposição à radiação UV e condensação foi realizado por meio de um sistema microcontrolado desenvolvido com a plataforma Arduino. Amostras compostas por uma matriz polimérica de borracha de silicone RTV (room temperature vulcanized) e preenchidos com alumina trihidratada (ATH) e cera de carnaúba foram submetidas ao envelhecimento acelerado na câmara desenvolvida. Os efeitos provocados pela exposição à radiação UV foram avaliados por meio de variações na hidrofobicidade e dureza das amostras.

\section{FUNDAMENTAÇÃO TEÓRICA}

\subsection{Envelhecimento por radiação UV}

A radiação ultravioleta é a parte do espectro eletromagnético que possui comprimentos de onda entre 100 e $400 \mathrm{~nm}$ e corresponde a cerca de $7 \%$ da energia emitida pelo Sol (INPE, 2021). A radiação UV pode ser subdividida em três faixas de comprimento de acordo com os efeitos fotobiológicos e a intensidade em que é absorvida pela atmosfera: UVC (100-280 nm), UVB (280-320 nm) e UVA (320-400 nm).

A radiação UVC corresponde a cerca de $1 \%$ da radiação solar e não atinge a superfície terrestre, pois é totalmente absorvida pelas camadas mais altas da atmosfera. A radiação UVB corresponde a cerca de 1,5\% da emissão solar e sofre grande atenuação pela camada de ozônio e outros constituintes da atmosfera, atingindo a superfície terrestre com baixa intensidade. A radiação UVA é menos afetada pela atmosfera e representa a maior parte da radiação ultravioleta que atinge a superfície terrestre (LOPO et al., 2013).

A energia espectral é inversamente proporcional ao comprimento de onda da radiação eletromagnética conforme a Lei de Planck, Equação 1. Assim, a radiação UVC possui maior energia na faixa espectral da radiação ultravioleta e pode provocar maiores danos ao material polimérico. Já a radiação UVA possui maior irradiância na faixa de ultravioleta e atinge mais rapidamente a superfície terrestre.

$$
E=h \times c / \lambda
$$

que $E$ é a energia fundamental, $h$ é a constante de Planck, $c$ é a velocidade da luz no vácuo e $\lambda$ é o comprimento de onda.

O envelhecimento por radiação UV pode ser realizado, basicamente, por meio da exposição em ambiente natural ou simulado em laboratório (artificial). A exposição no ambiente natural expõe as amostras à situação 
real de uso, reproduzindo fielmente a atuação dos diversos constituintes ambientais (temperatura, umidade, radiação UV, poluentes, entre outros). Entretanto, este procedimento requer um longo período de duração e a reprodutibilidade é dificultada devido às variações das condições geográficas e climáticas (FECHINE; SANTOS; RABELLO, 2006; SARON, 2001).

O envelhecimento artificial por radiação UV permite simular situações que provocam a degradação dos polímeros de forma acelerada pela exposição à radiação luminosa. Para tanto, a incidência solar pode ser simulada em laboratório por meio de lâmpadas fluorescentes especiais ou de arco de xenônio, que emitem radiação na faixa espectral do ultravioleta. Outros parâmetros como temperatura e umidade, devem ser considerados durante a realização dos testes para proporcionar maior representatividade e reproduzir as condições ambientais de forma mais realística (FECHINE; SANTOS; RABELLO, 2006; SARON, 2001).

Existe uma grande dificuldade em estabelecer uma correlação entre os sistemas de envelhecimento natural e artificial em função do tempo de exposição. A causa está associada a grande variabilidade dos elementos da natureza que influenciam no desempenho do polímero. Uma correlação aproximada entre o envelhecimento natural e artificial pode ser visualizada na Figura 1,(BRANDALISE, 2008, RIOS, 2012, ADEXIMCONEXIM, 2000).

Figura 1: Correlação entre degradação acelerada e degradação natural.

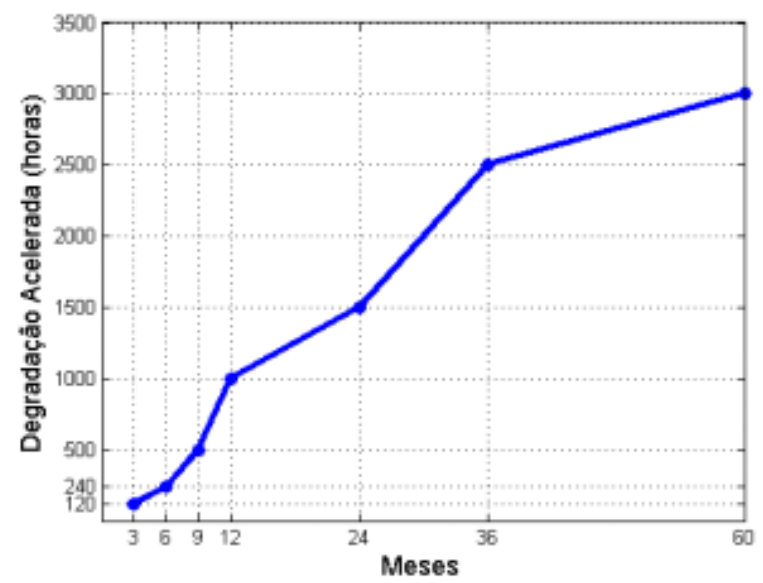

Fonte: Adaptado de ADEXIM-CONEXIM 2000).

\subsection{Aspectos construtivos baseados na ASTM G 53}

A norma ASTM G53 (AMERICAN SOCIETY FOR TESTING AND MATERIALS, 1998) descreve os princípios básicos e procedimentos operacionais do arranjo para simular a deterioração causada pela luz solar e umidade proveniente da chuva ou orvalho. No entanto, esta norma não especifica as condições de exposição que o material deve ser submetido durante o teste.

Basicamente, o arranjo é formado por uma câmara de teste contendo lâmpadas UV e um sistema de condensação. A câmara de teste deve ser construída de material resistente à corrosão e abrigar oito lâmpadas fluorescentes UV, um reservatório de água aquecida, suporte para as amostras que serão testadas e dispositivos para controlar e indicar os tempos de operação e temperaturas. As lâmpadas UV são divididas igualmente em dois compartimentos compostos por quatro lâmpadas para a realização de ensaios de amostras.

Dois tipos de configurações (A e B) são especificados na norma a depender da distância entre as amostras de testes e as lâmpadas UV, conforme mostrado na Figura 2. Para a configuração A (Figura 2a) as superfícies de testes das amostras são dispostas em um plano paralelo ao plano das lâmpadas, com uma distância constante de $50 \mathrm{~mm}$ entre os planos e espaçamento de 70 mm entre os centros das lâmpadas. Já para a configuração B (Figura 2p), a distância entre as amostras e as lâmpadas, assim como o espaçamento entre as lâmpadas, são variáveis.

O sistema de condensação no arranjo consiste na geração de vapor resultante do aquecimento de um reservatório de água que se estende sob toda a área da amostra. O reservatório deve conter água com no mínimo de $25 \mathrm{~mm}$ de profundidade e o fornecimento deve ser realizado empregando um controle automático para regular o nível da água no reservatório.

O arranjo deve possuir termômetros para medição da temperatura nas amostras e temporizadores de ciclos para programar os períodos de exposição à radiação UV e de condensação. Além disso, o arranjo deve possuir um relógio para medição do tempo total de operação e de exposição à radiação UV.

\section{METODOLOGIA}

\subsection{Material}

Para o desenvolvimento do equipamento foram utilizados os seguintes materiais:

- chapas de alumínio (2 mm x 2000 mm x 1000 $\mathrm{mm})$; 


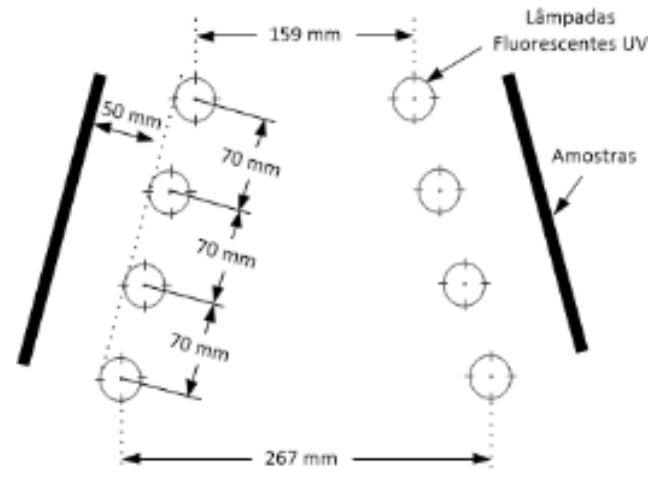

(a)

Fonte: Adaptado de ASTM G. 53 (1998).

- lâmpadas UVB Philips broadband TL 40W/12;

- reatores eletrônicos EL1/254a26 - Philips;

- reservatórios de metal resistente à corrosão $(27 \mathrm{~cm}$ $\mathrm{x} 19 \mathrm{~cm} \times 5 \mathrm{~cm})$;

- resistências de imersão tipo $\mathrm{U}$ de $1 \mathrm{~kW}$;

- bomba vibratória ULKA modelo E, tipo EX5;

- chip ATmega328P;

- módulo relé;

- chaves magnéticas (reed switches);

- termopar tipo K com módulo MAX6675;

- display LCD 20x4;

- módulo serial I2C;

- teclado matricial $4 \times 4$;

- disjuntor termomagnético (Siemens B16 5sx1 220/380V);

- botões interruptores;

- bola de isopor (10mm de diâmetro);

- tubos e conexões;

- registro de água;

- ímã;

- reservatório de água externo.

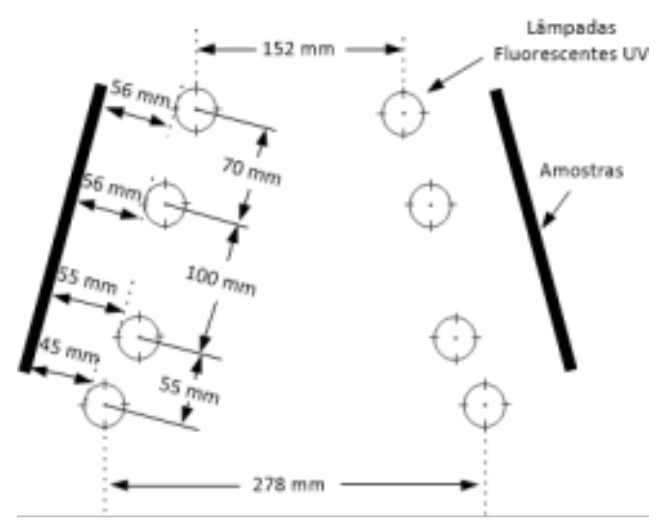

(b)

\subsection{Aspectos construtivos do equipamento}

\subsubsection{Estrutura do equipamento}

Uma ilustração da estrutura da câmara de envelhecimento pode ser visualizada na Figura 3 . A estrutura foi construída com chapas de alumínio, que são resistentes à corrosão.

$\mathrm{O}$ equipamento projetado consiste em uma simplificação da configuração Tipo A proposta pela norma ASTM G53, indicada na seção 3. No projeto foi considerado apenas um dos compartimentos do arranjo, permitindo a instalação de quatro lâmpadas UV tubulares. Além disso, o equipamento é formado por um sistema de condensação e um sistema de controle.

As lâmpadas e as amostras são dispostas no compartimento de cor verde da Figura 3. As lâmpadas são espaçadas entre si de $70 \mathrm{~mm}$ e o plano que as contém encontra-se inclinado em relação ao plano vertical e a uma distância de $50 \mathrm{~mm}$ das amostras.

Um termopar foi instalado na parte superior do compartimento para monitoramento da temperatura. Uma tampa de acesso foi instalada na parte frontal do equipamento com o objetivo de manter a câmara hermeticamente fechada e impedir riscos de exposição do operador a radiação UV.

Os reservatórios de água utilizados para realizar o aquecimento da água e condensação no interior do equipamento foram instalados na parte inferior da estrutura (indicados pela cor cinza na Figura 3 ).

As partes laterais da câmara, indicadas pela cor amarela da Figura 3, são compostas por cavidades com tampa e destinadas a instalação e isolação dos contatos elétricos das lâmpadas UV e das resistências de imersão dispostas nos reservatórios. 


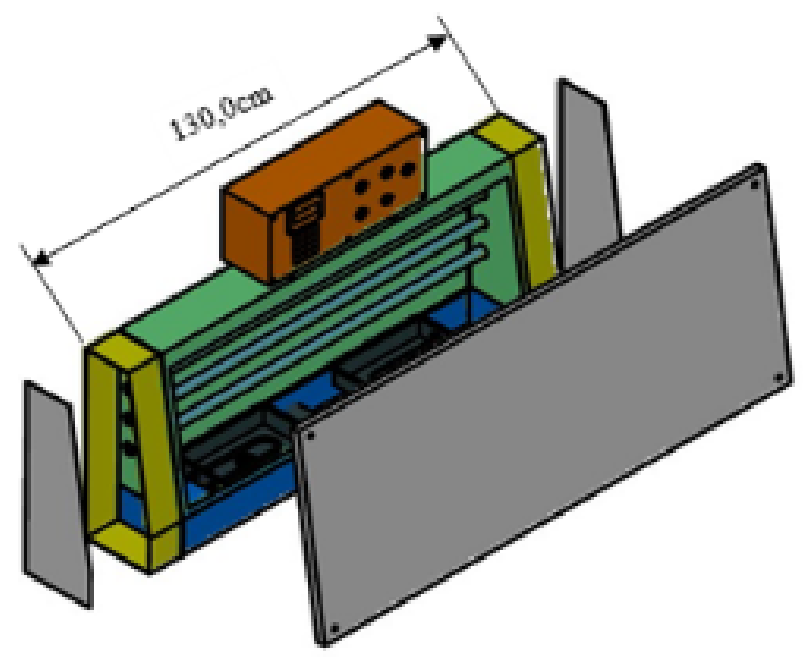

(a)

O sistema de controle responsável pela programação e monitoramento do ensaio encontra-se instalado na parte superior do equipamento, indicado pela cor marrom da Figura 3 .

\subsubsection{Sistema de condensação}

O sistema de condensação desenvolvido consiste, basicamente, em dois reservatórios de metal contendo uma resistência de imersão tipo $\mathrm{U}$ de $1 \mathrm{~kW}$ instalada em cada, uma bomba d'agua e chaves magnéticas (reed switches) para controle do nível de água.

Os reservatórios de água são instalados na parte inferior da estrutura (indicados pela cor cinza na Figura 3 a) e interconectados por uma tubulação metálica. Além disso, os reservatórios encontram-se conectados a uma tubulação de PVC para água quente em forma de U localizada na parte posterior do equipamento e destinada ao monitoramento e regulação do nível de água. O esquema do sistema de condensação pode ser visualizado na Figura 4

Uma bomba d'agua foi instalada na parte posterior da estrutura e conectada a um dos terminais da tubulação em forma de $U$ para permitir o fornecimento de água para os reservatórios. A água é bombeada a partir de um reservatório externo. O procedimento para acionamento da bomba e regulação do nível de água no reservatório é baseado no funcionamento dos sensores de nível A (nível superior) e B (nível inferior), constituídos por chaves magnéticas (reed switches) e instalados no outro terminal da tubulação em forma de U, conforme mostrado na Figura 5

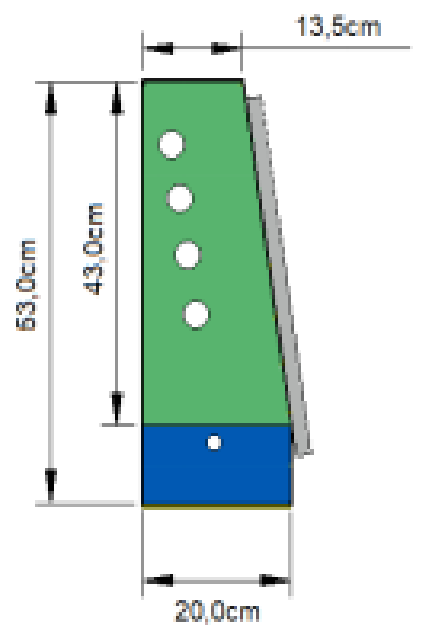

(b)

Figura 4: Esquema do sistema de condensação.

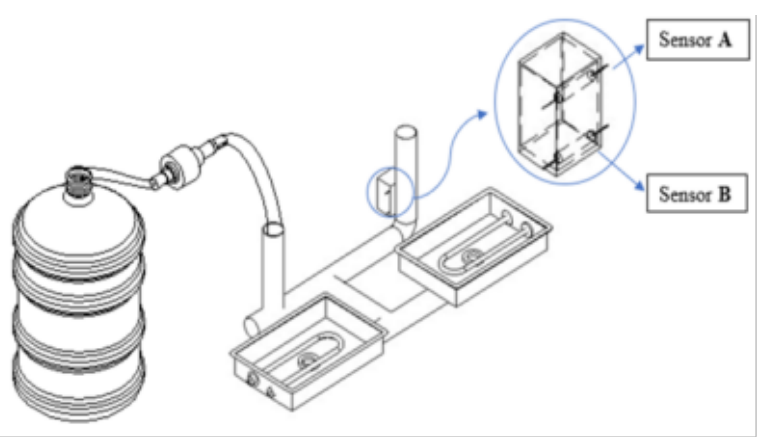

$\mathrm{O}$ acionamento dos sensores $\mathrm{A}$ e $\mathrm{B}$, instalados na parede da tubulação, é realizado por meio da detecção de um ímã introduzido em um material flutuante, resistente a altas temperaturas e que foi inserido na tubulação. Quando o nível da coluna de água encontra-se em um nível crítico, ocorre o acionamento do sensor B e a energização da bomba, conforme ilustrado na Figura 5 (a). Quando a coluna de água atinge o nível máximo é realizado o acionamento do sensor A e o desligamento da bomba, conforme ilustrado na Figura 5 (b). Os sensores estão conectados ao sistema de controle microcontrolado que realiza o acionamento de um relé responsável pela energização/desenergização da bomba.

\subsubsection{Sistema de controle}

O sistema de controle da câmara de envelhecimento é responsável pelo acionamento das lâmpadas, moni- 


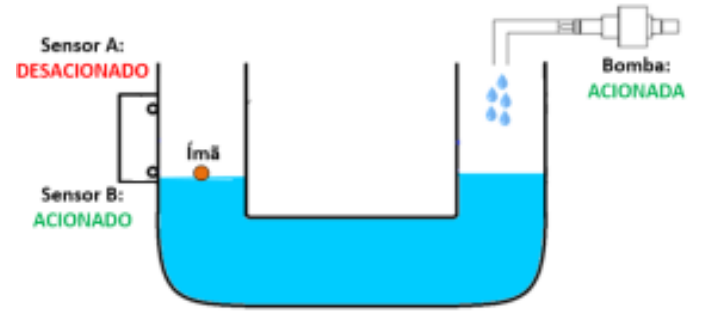

(a)

toramento da temperatura interna, acionamento das resistências de imersão, controle do nível de água do sistema de condensação e controle dos ciclos de exposição ao longo do ensaio. O esquema do sistema de controle pode ser visualizado na Figura 6.

O sistema de controle foi desenvolvido por meio da utilização do Arduino UNO, que utiliza o microcontrolador ATmega328P, possibilitando flexibilidade e baixo custo para o desenvolvimento do sistema. A configuração do ensaio é realizada pelo operador por meio de um teclado matricial e as informações da programação e monitoramento dos parâmetros do ensaio podem ser visualizadas por meio de um display LCD. Para a conexão do LCD ao Arduino foi utilizado um módulo I2C com o objetivo de reduzir a quantidade de entradas necessárias para a conexão.

Para a configuração do ensaio devem ser informados: a duração total do ensaio e dos ciclos de exposição à radiação UV e condensação. Além disso, deve ser informada a temperatura do ciclo de condensação.

A temperatura no interior da câmara é monitorada por meio de um termopar conectado ao microcontrolador e indicada no LCD. Durante os ciclos de condensação, a temperatura medida pelo termopar é comparada com o valor pré-definido pelo operador. Para valores abaixo da temperatura informada pelo operador, as resistências de imersão são energizadas por meio do relé R1 para possibilitar o aquecimento da água dos reservatórios. Ao atingir a temperatura de referência, as resistências são desenergizadas. Adicionalmente, uma chave de segurança (interruptor S1) foi instalada e permite realizar o desligamento manual da resistência caso seja observado algum problema ou por necessidade do operador.

As chaves magnéticas (reed switch) são utilizadas para o monitoramento do nível de água dos reservatórios. Para níveis abaixo de um ponto crítico, a chave magnética de nível inferior é fechada, ocasionando o acionamento do relé R2 e a bomba é energizada para injetar água no reservatório. A bomba permanece ener-

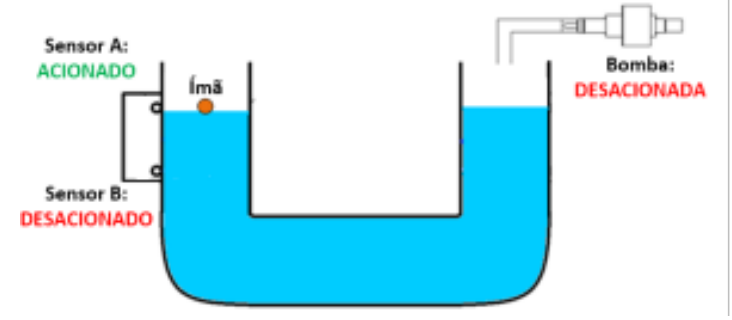

(b)

gizada até atingir o nível de água desejado no reservatório. Ao atingir o nível de água desejado, a chave de nível superior é fechada, ocasionando o desacionamento do relé $\mathrm{R} 2$ e a bomba é desenergizada, interrompendo o fornecimento de água. O interruptor S2 permite realizar o desligamento manual da bomba d'água.

Para o ciclo de exposição à radiação UV, dois reatores eletrônicos são acionados por meio dos relés R3 e R4 para realizar a ignição das lâmpadas. Cada reator eletrônico realiza o acionamento de duas lâmpadas UV. As chaves $\mathrm{S} 3$ e S4 permitem selecionar manualmente a quantidade e/ou posição das lâmpadas que serão utilizadas no ensaio.

O Arduino, o LCD, o teclado, o módulo relé, o módulo MAX6675 do termopar, os interruptores e os reatores, indicados na Figura 6, foram instalados em um painel, conforme mostrado na Figura 7. Um disjuntor foi adicionado na parte interna do painel para permitir a proteção contra sobrecorrentes no equipamento. Além disso, uma chave geral S0 foi instalada para realizar a energização/desenergização do equipamento.

\subsection{Aplicação do equipamento}

O equipamento foi utilizado no envelhecimento de quatro compósitos formados por uma matriz polimérica de borracha de silicone RTV (room temperature vulcanized) e preenchidos com alumina tri-hidratada (ATH) e cera de carnaúba em diferentes concentrações mássicas. Para cada composição foi produzida uma amostra com dimensões $50 \mathrm{~mm}$ x $120 \mathrm{~mm}$ x $6 \mathrm{~mm}$.

As amostras foram submetidas ao envelhecimento acelerado na câmara desenvolvida por um período de 500 horas $(\approx 21$ dias). O ensaio foi realizado em ciclos com duração de 10 horas, subdivididos em 8 horas de exposição à radiação UV e 2 horas de condensação visando simular, respectivamente, os efeitos de degradação causados pela luz solar e do orvalho na superfície das amostras.

A avaliação das amostras foi feita a partir do moni- 


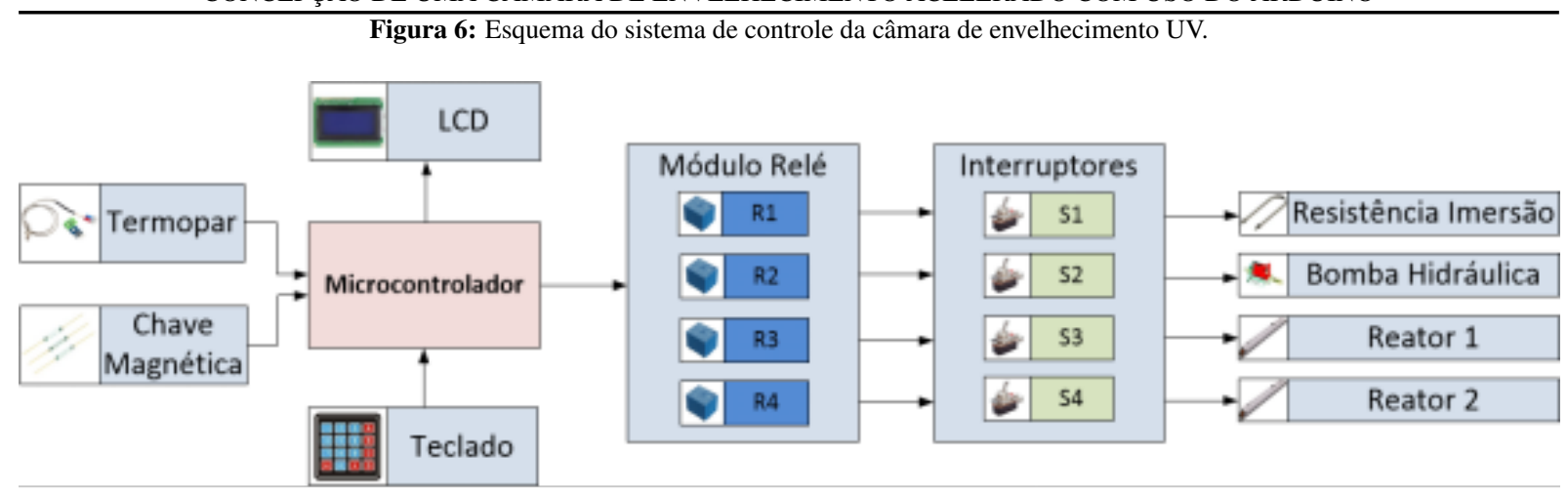

Figura 7: Painel de controle da câmara de envelhecimento UV.

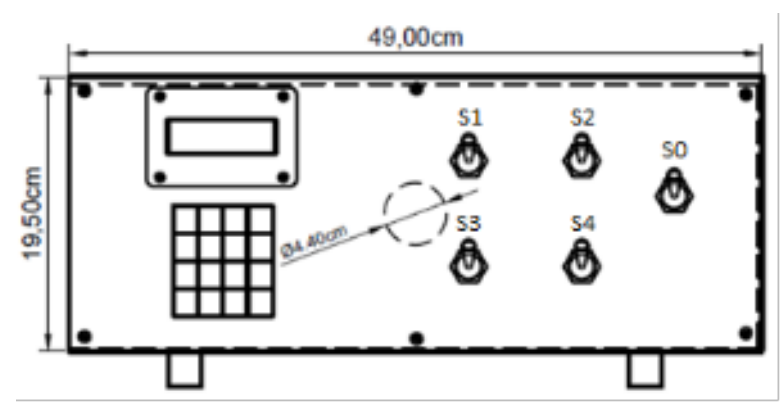

toramento da hidrofobicidade e medição da dureza antes e após a realização do ensaio no equipamento. O monitoramento da hidrofobicidade é bastante utilizado em diversas análises de revestimentos, pois permite avaliar o nível de repulsão de um líquido sobre uma superfície. No sistema elétrico, por exemplo, a hidrofobicidade em polímeros aplicados no isolamento externo confere maiores níveis de suportabilidade elétrica (SUWARNO; PRATOMOSIWI, 2009, JAMALUDIN et al., 2017; LI et al., 2017). Já a medição da dureza em polímeros pode fornecer informações do grau de cura e permite identificar propriedades mecânicas afetadas por mudanças na composição química, macroestrutural e envelhecimento (FISCHER-CRIPPS, 2004).

Para o monitoramento da hidrofobicidade foi empregado o método do ângulo de contato estático realizado a partir do arranjo proposto por Nóbrega (2016). $\mathrm{O}$ valor do ângulo de contato consistiu na média da medição do ângulo de cinco gotas aplicadas em áreas adjacentes da amostra. A dureza das composições poliméricas foi determinada com o auxílio de um durômetro portátil Shore tipo A, sendo o valor da dureza para cada instante de medição dada pela média de três medições efetuadas em diferentes regiões da amostra analisada.

\section{Resultados}

\section{1}

Equipamento desenvolvido

Na Figura 8 é mostrada uma fotografia da estrutura da câmara desenvolvida para a realização de testes de envelhecimento acelerado por meio de exposição à radiação UV, umidade e temperatura.

Figura 8: Vista frontal da estrutura da câmara de envelhecimento desenvolvida.

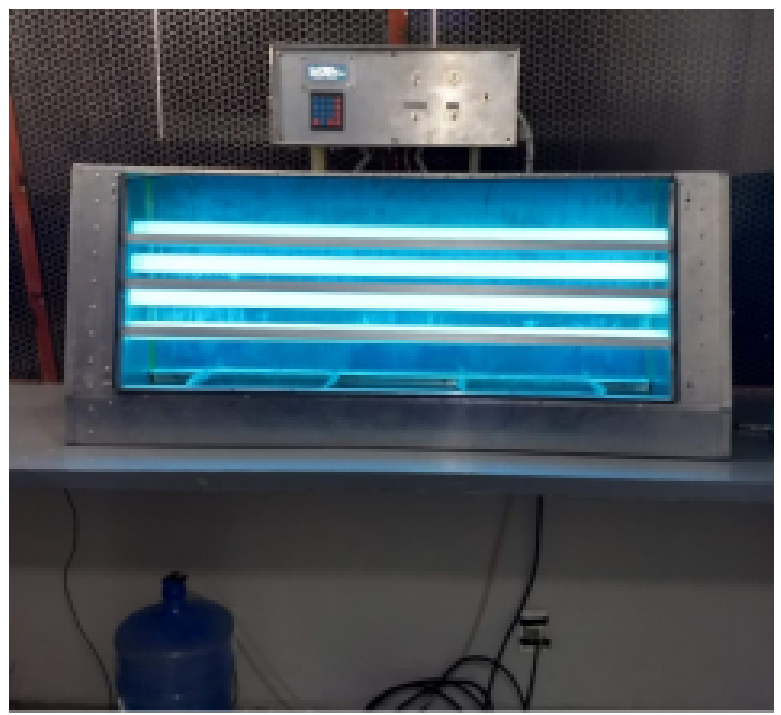

Na Figura 9 é mostrado o sistema de condensação desenvolvido antes da instalação no equipamento. $\mathrm{O}$ sistema permite simular condições de umidade e temperatura durante ensaios de envelhecimento acelerado.

Inicialmente, o sistema de condensação foi avaliado isoladamente com o objetivo de verificar o correto funcionamento dos sensores e da lógica de programação implementada, além do fluxo de água inserido nos reservatórios após a evaporação promovida pelas resistên- 


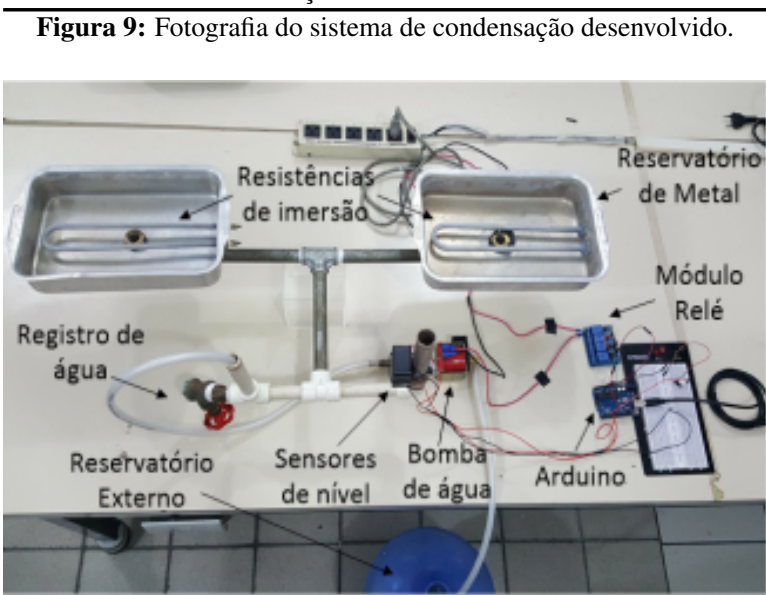

cias de imersão e eventuais problemas de conexão e vedação.

Posteriormente, o sistema de condensação foi integrado à estrutura da câmara de envelhecimento acelerado desenvolvida e sua lógica de programação foi implementada no código principal de funcionamento do equipamento. Constatou-se que a plataforma Arduino foi satisfatória no controle dos sistemas de acionamento das lâmpadas e de condensação, além de proporcionar o efetivo monitoramento dos sensores e funcionamento adequado dos módulos responsáveis pela interface com o usuário para configuração do equipamento.

\subsection{Hidrofobicidade}

Na Figura 10 são mostrados os valores de ângulo de contato medidos para cada amostra antes do início e após o término de exposição à radiação UV.

Figura 10: Valores de ângulo de contato estático para as amostras investigadas.

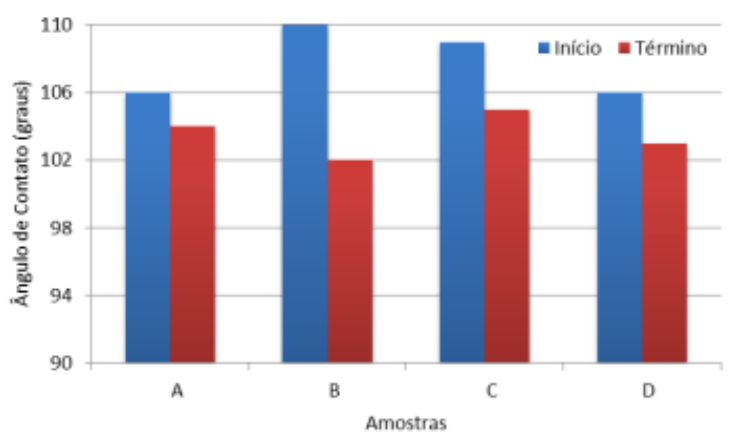

Analisando a Figura 10 constata-se uma redução nos valores de ângulo de contato para amostras expostas à radiação UV. Antes do processo de envelhecimento, as amostras tinham valores de ângulo de contato entre $106^{\circ}$ e $110^{\circ}$. Após exposição à radiação UV, as amostras possuíam valores entre $102^{\circ}$ e $105^{\circ}$. A redução nos valores de ângulo de contato evidencia indícios de degradação das amostras, conforme Yi, Shi e Liang (2014), Nazir e Phung (2016).

\subsection{Dureza}

Na Figura 11 são mostrados valores de dureza Shore A para as amostras investigadas.

Figura 11: Valores de dureza Shore A para as amostras investigadas.

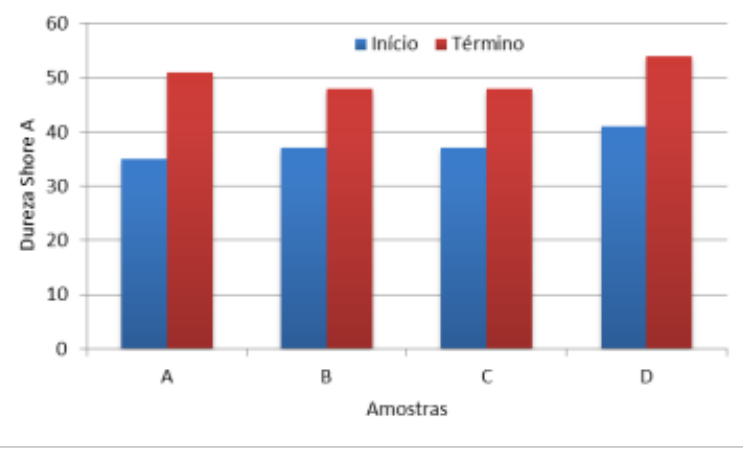

Quando se analisa a Figura 11, evidenciam-se alterações nas propriedades mecânicas provocadas pela radiação UV a partir do aumento dos valores de dureza do polímero. Este fato pode ser utilizado como parâmetro para evidenciar o fenômeno de envelhecimento, conforme Wang et al. (2016), Nazir e Phung (2016). Antes do processo de envelhecimento, as amostras tinham valores de dureza variando entre $35 \mathrm{HA} \mathrm{e} 41 \mathrm{HA}$. Após exposição à radiação UV, as amostras possuíam valores variando entre $48 \mathrm{HA}$ e $54 \mathrm{HA}$.

\section{Considerações finais}

Neste trabalho foi apresentado a concepção e o desenvolvimento de um equipamento capaz de promover a degradação de materiais poliméricos por meio da exposição à radiação UV, temperatura e umidade.

O equipamento proposto permite a instalação de até quatro lâmpadas de ultravioleta e os sistemas de condensação e controle podem ser obtidos a partir de componentes e materiais disponíveis no comércio. Os princípios adotados para o projeto e desenvolvimento da câmara de envelhecimento acelerado foram baseados em requisitos das normas ASTM G53, ASTM G151 e ASTM G154 e com custos de construção reduzidos frente aos modelos disponíveis no mercado. A automação e o controle do equipamento foram realizados por meio da plataforma Arduino, que possui código aberto 
CONCEPÇÃO DE UMA CÂMARA DE ENVELHECIMENTO ACELERADO COM USO DO ARDUINO

e possibilita a reprodução em outras instituições de ensino e pesquisa.

A utilização da plataforma Arduino mostrou-se satisfatória no controle dos sistemas de acionamento das lâmpadas e de condensação do equipamento desenvolvido. Além disso, o Arduino proporcionou o efetivo monitoramento dos sensores e uma comunicação adequada com demais periféricos como teclado e LCD.

Os resultados do monitoramento da hidrofobicidade e medição da dureza de amostras poliméricas evidenciaram alterações nas propriedades dos compósitos, indicando o envelhecimento das amostras após exposição à radiação UV e a aplicabilidade do equipamento proposto neste trabalho.

Portanto, o desenvolvimento da câmara de envelhecimento acelerado proposto nesse trabalho possibilita a difusão do projeto/metodologia de um equipamento automatizado e com custos reduzidos, capaz de auxiliar em pesquisas relacionadas ao estudo e envelhecimento de materiais. Ainda, o desenvolvimento do equipamento pode proporcionar ganhos de autonomia de laboratórios que desenvolvem atividades de ensino, pesquisa e extensão, possibilitando a ampliação das pesquisas e a prestação de serviços.

Como trabalho futuros, o equipamento pode ser aperfeiçoado a partir da instalação de radiômetros e dispositivos que permitam controlar a intensidade da irradiação emitida pelas lâmpadas. Além disso, espera-se adaptar a tecnologia proposta para dispositivos móveis com o propósito de permitir o acompanhamento do ensaio à distância e/ou o acesso remoto ao equipamento.

\section{Agradecimentos}

Os autores agradecem a Universidade Federal de Campina Grande (UFCG), em especial ao Laboratório de Alta Tensão (LAT) pela disponibilização do ambiente, material e assistência para desenvolvimento do equipamento.

Os autores agradecem ao Conselho Nacional de Desenvolvimento Científico e Tecnológico (CNPq) por fomentar esta pesquisa.

\section{REFERÊNCIAS}

ADEXIM-CONEXIM. Correlação entre o tempo real de intemperismo e a ação do sistema C-UV com base na ASTM G53/154. 2000.

\author{
ASTM G. 151. Standard Practice for Exposing \\ Nonmetallic Materials in Accelerated Test \\ Devices that Use Laboratory Light Sources. \\ West Conshohocken: AMERICAN SOCIETY FOR \\ TESTING AND MATERIALS, 2000.
}

ASTM G. 154. Operating Fluorescent Light Apparatus for UV Exposure of Nonmetallic Materials. West Conshohocken: AMERICAN SOCIETY FOR TESTING AND MATERIALS, 2016.

ASTM G. 53. Standard Practice for Operating

Light-and Water-Exposure Apparatus (Fluorescent

UV-Condensation Type) for Exposure of nonmetallic Materials. West Conshohocken: AMERICAN SOCIETY FOR TESTING AND MATERIALS, 1998.

BRANDALISE, R. N. Avaliação da degradação biótica e abiótica da mistura polimérica entre de polietileno de alta densidade com poli (álcool vinilico). 2008. Tese (Doutorado em Engenharia de Materiais) - Universidade Federal do Rio Grande do Sul, Porto Alegre, 2008.

FECHINE, G. J. M.; SANTOS, J. A. B. d.; RABELLO, M. S. Avaliação da fotodegradação de poliolefinas através de exposição natural e artificial. Química Nova, SciELO Brasil, v. 29, n. 4, p. 674-680, 2006.

FISCHER-CRIPPS, A. C. A simple phenomenological approach to nanoindentation creep. Materials Science and Engineering: A, Elsevier, v. 385, n. 1, p. 74-82, 2004.

INPE. Radiação UV. 2021. Disponível em: <http://satelite.cptec.inpe.br/uv/> Acesso em: out. 2021.

JAMALUDIN, F. A.; AB-KADIR, M. Z. A.; IZADI, M.; AZIS, N.; JASNI, J.; ABD-RAHMAN, M. S. Effects of rtv coating on the electrical performance of polymer insulator under lightning impulse voltage condition. PloS one, Public Library of Science San Francisco, CA USA, v. 12, n. 11, p. e0187892, 2017.

LI, Y.; JIN, H.; NIE, S.; ZHANG, P.; GAO, N. Dynamic behavior of water droplets and flashover characteristics on a superhydrophobic silicone rubber surface. Applied Physics Letters, AIP Publishing LLC, v. 110, n. 20, p. 201602, 2017.

LOPO, A. B.; SPYRIDES, M. H. C.; LUCIO, P. S.; SIGRÓ, J. Radiação ultravioleta, ozônio total e aerossóis na cidade de natal-rn. Holos, Instituto Federal de Educação, Ciência e Tecnologia do Rio Grande do Norte, v. 6, n. 1, p. 3-21, 2013.

NAZIR, M. T.; PHUNG, B. Ultraviolet weathering resistance performance of micro/nano silica filled silicone rubber composites for outdoor insulation. In: 2016 International Conference on Condition 
Monitoring and Diagnosis (CMD). Xi'an, China: IEEE, 2016. p. 1035-1038.

NEKEB, A.; HADDAD, A.; HARID, N.; GRIFFITHS, H.; WATERS, R. Effects of uv irradiation on textured silicone rubber material. In: 47th International Universities Power Engineering Conference (UPEC). Uxbridge, UK: IEEE, 2012. p. 1-5.

NóBREGA, R. B. Avaliação de uma Nova Carga na Composição de Revestimentos Externos Aplicados em Isoladores de Alta Tensão. Dissertação (Mestrado) - Universidade Federal de Campina Grande, Campina Grande, 2016.

RABELLO, M. S. Aditivação de polímeros. 1. ed. São Paulo: Artliber, 2000.

RIOS, A. S. Estudo do envelhecimento acelerado de materiais compósitos revestidos com poliuretano aplicados em aerogeradores. Dissertação (Mestrado) — Universidade Federal do Ceará, Fortaleza, 2012.

SARON, C. Estudo da degradação térmica e fotoquímica das blendas PPO/HIPS. Dissertação (Mestrado) - Universidade Estadual de Campinas, Campinas, 2001.

SUWARNO; PRATOMOSIWI, F. Application of rtv silicone rubber coating for improving performances of ceramic outdoor insulator under polluted dondition. In: 2009 International Conference on Electrical Engineering and Informatics. Bangi, Malaysia: IEEE, 2009. v. 2, p. 581-587.

ULLAH, I.; AMIN, M.; NAZIR, M. T.; HUSSAIN, $\mathrm{H}$. Impact of accelerated ultraviolet weathering on polymeric composite insulators under high voltage dc stress. CSEE Journal of Power and Energy Systems, CSEE, v. 1, n. 1, p. 1-11, 2020. ISSN 2096-0042.

WANG, Z.; ZHANG, X.; WANG, F.; LAN, X.; ZHOU, Y. Effects of aging on the structural, mechanical, and thermal properties of the silicone rubber current transformer insulation bushing for a $500 \mathrm{kv}$ substation. SpringerPlus, SpringerOpen, v. 5, n. 1, p. 1-6, 2016.

YI, C. F.; SHI, Q.; LIANG, P. S. Effect of uv radiation on htv-silicon rubber under different humidity. Advanced Materials Research, Trans Tech Publ, v. 986, n. 1, p. 55-58, 2014. 